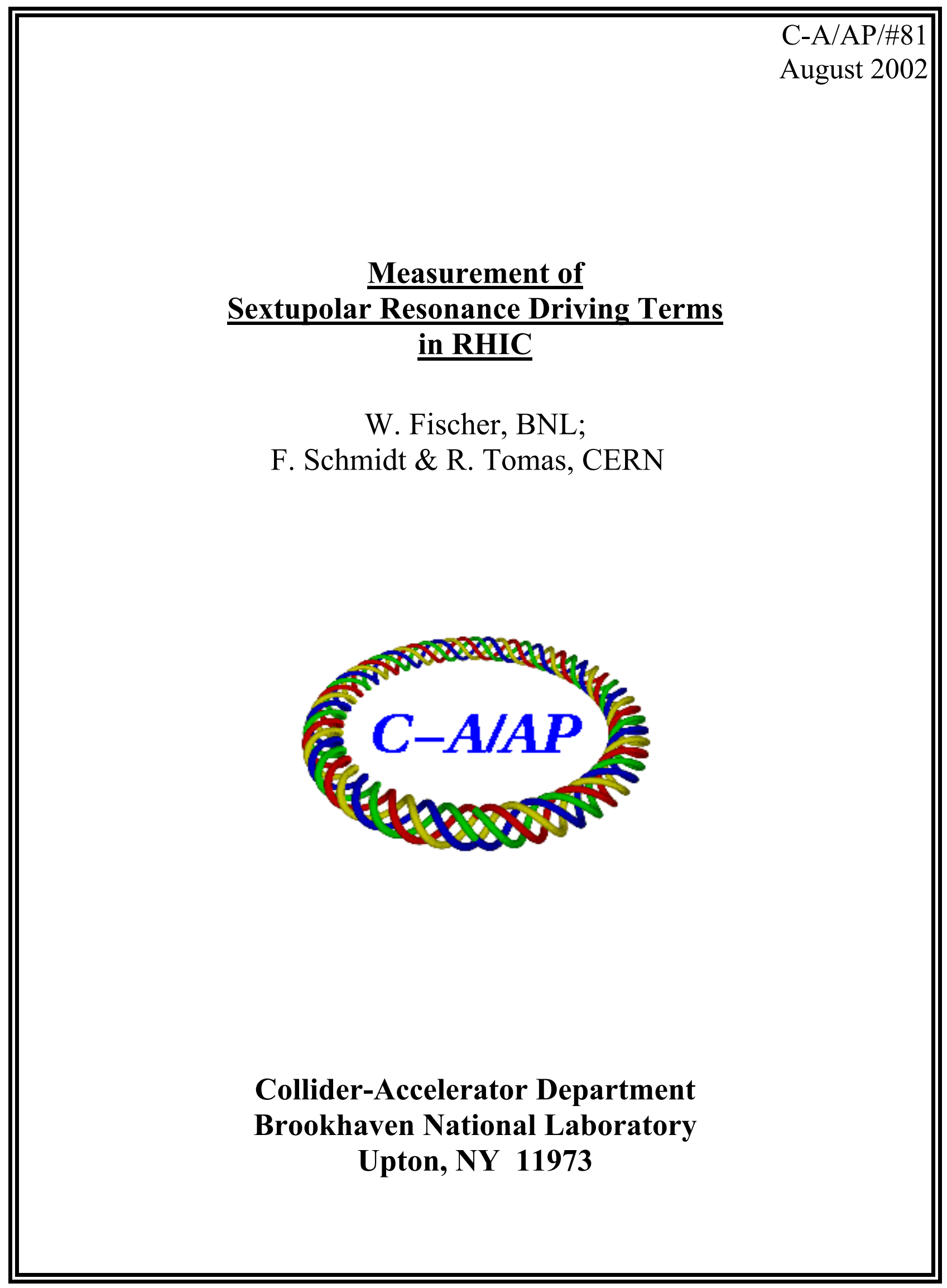




\title{
Measurement of Sextupolar Resonance Driving Terms in RHIC
}

\author{
W. Fischer, BNL, F. Schmidt and R. Tomás, CERN
}

30 August, 2002

\begin{abstract}
Theory predicts that resonance driving terms can be determined by harmonic analysis of BPM data recorded after applying single kicks. In recent experiments at the CERN SPS this technique has been succesfully applied to measure coupling and sextupolar resonance terms around the ring. A similar experiment has been carried out in RHIC, BNL, to proove the feasibility of this measurement in this more complex machine. Results of the experiment are presented, including a direct measurement of sextupolar resonances and a comparison to the model.
\end{abstract}

\section{Introduction}

The turn-by-turn single particle motion in normalised coordinates to first order in the non-linearities is given by [1]

$$
\begin{aligned}
\hat{x}(N)- & i \hat{p}_{x}(N)=\sqrt{2 I_{x}} e^{i\left(2 \pi \nu_{x} N+\psi_{x_{0}}\right)} \\
& -2 i \sum_{j k l m} j f_{j k l m}\left(2 I_{x}\right)^{\frac{j+k-1}{2}}\left(2 I_{y}\right)^{\frac{l+m}{2}} \\
& \times e^{i\left[(1-j+k)\left(2 \pi \nu_{x} N+\psi_{x_{0}}\right)+(m-l)\left(2 \pi \nu_{y} N+\psi_{y_{0}}\right)\right]}
\end{aligned}
$$

where $I_{x}$ and $I_{y}$ are the horizontal and vertical actions, $\psi_{x_{0}}$ and $\psi_{y_{0}}$ are the horizontal and vertical initial phases, $\nu_{x}$ and $\nu_{y}$ are the horizontal and vertical tunes including the amplitude dependent detuning and the factors $f_{j k l m}$ are the generating function terms. These are related to the Hamiltonian terms $h_{j k l m}$ by the following expression,

$$
f_{j k l m}=\frac{h_{j k l m}}{1-e^{-i 2 \pi\left[(j-k) \nu_{x}+(l-m) \nu_{y}\right]}} .
$$

Note that the term $f_{j k l m}$ drives the resonance $(j-k, l-m)$. The Hamiltonian terms are defined by the following expansion of the non-linear Hamiltonian,

$$
\begin{aligned}
H= & \sum_{j k l m} h_{j k l m}\left(2 I_{x}\right)^{\frac{j+k}{2}}\left(2 I_{y}\right)^{\frac{l+m}{2}} \\
& \times e^{-i\left[(j-k)\left(\psi_{x}+\psi_{x_{0}}\right)+(l-m)\left(\psi_{y}+\psi_{y_{0}}\right)\right]},
\end{aligned}
$$


where $\psi_{x}$ and $\psi_{y}$ are the horizontal and vertical angle variables. Eqs. (1) and (2) suggest that a FFT of the turn-by-turn complex signal can be used to measure the generating function and the Hamiltonian terms. The spectral line $(1-j+k, m-l)$ depends only on the term $f_{j k l m}$. By line $(m, n)$ we mean the spectral line with frequency $m \nu_{x}+n \nu_{y}$. To illustrate these relations the third order resonance is studied. The Hamiltonian and the generating function terms driving the third order resonance are $h_{3000}$ and $f_{3000}$ respectively. The spectral line produced in the horizontal motion by this resonance is therefore ($2,0)$. In a real machine the complex signal is constructed from two pick-ups with $90^{\circ}$ phase advance. If the phase advance between the two pick-ups is not exactly $90^{\circ}$ a linear transformation can be applied to the data from both pick-ups to obtain the corresponding set of data with $90^{\circ}$ of phase advance. Only one pick-up cannot be used to measure resonance driving terms unambiguously since the spectral lines $(m, n)$ and $(-m,-n)$ cannot be disentangled.

The Hamiltonian and the generating function terms depend on the longitudinal location where they are calculated. To understand how they vary along the ring the values of a Hamiltonian term at both sides of a source of non-linearity are compared. Prior to this element the term is $h_{j k l m}^{1}$ and after it is $h_{j k l m}^{2}$. The non-linearity contributes to the first case with the quantity $k_{j k l m}$ and to the second case with the quantity $e^{-i 2 \pi\left[(j-k) \nu_{x}+(l-m) \nu_{y}\right]} k_{j k l m}$ because the element has moved to the end of the lattice. Therefore the relation between the two Hamiltonian terms is expressed as

$$
h_{j k l m}^{2}=h_{j k l m}^{1}+\left(e^{-i 2 \pi\left[(j-k) \nu_{x}+(l-m) \nu_{y}\right]}-1\right) k_{j k l m},
$$

the equivalent relation between the generating function terms is given by

$$
f_{j k l m}^{2}=f_{j k l m}^{1}-k_{j k l m} .
$$

These relations state that the amplitude of these terms changes abruptly at the location of the sources. Their amplitudes remain constant along sections free of sources. This feature is very important since it allows the localisation of multipolar kicks. Applications of this feature in the CERN SPS can be found in [2] and [3].

In a real machine the beam is not a single particle but a particle distribution and processes like the beam decoherence change the Fourier spectrum of the turn-by-turn motion. The effect of the decoherence due to amplitude detuning has been described in [2]. The relevant conclusion is that the spectral line $(\mathrm{m}, 0)$ of a decohered signal is reduced by a factor of $|\mathrm{m}|$ compared to the single particle case. In particular, the two spectral lines $(-2,0)$ and $(2,0)$, produced by sextupolar fields, from the decohered motion are decreased by a factor of 2 compared to the single particle case. In order to compare the results from the experiment to single particle simulations the corresponding factor is applied to the experimental results. 


\section{Description of the RHIC model}

The tracking program SixTrack [6] is used for the analysis. The RHIC tracking model is constructed from an ideal lattice, characterized by a $\beta$-function of $10 \mathrm{~m}$ at all interaction points. This configuration was used in the experiment. Magnetic field errors are introduced in the arc dipoles and quadrupoles. At injection, these field errors dominate the dynamic aperture.

For the dipoles, cold measurements at $660 \mathrm{~A}$ in 58 magnets are used to determine the average and rms values for the geometric field errors $[4,5]$. The dipole current at injection is $470 \mathrm{~A}$. Normal and skew components are measured up to 22-poles. The measured mean and rms values are used to assign random errors in the lattice. Mean and rms values for the sextupole component, dominated by persistent currents, are determined in a separate measurement, and also used to assign random errors in the lattice.

For the quadrupoles, only geometric errors are considered. Mean and rms values are measured in 380 magnets at $30 \mathrm{~A}$ and room temperature. A good correlation is found between warm and cold measurements in these magnets [4, 5]. No additional persistent current error contributions are included in the quadrupoles.

Tunes and chromaticities are set to the values observed in the experiment. Closed orbit errors are disregarded. In the experiment one sextupole (RHIC site wide name yi7-sx3, lattice name $b 2 m 07 c 3 y$ ) was changed to excite the third order resonance.

\section{Description of the experiment}

The measurement of resonance driving terms was carried out in gold operation at injection. Relevant parameters are displayed in Tab. 1. In all cases the transverse injection oscillations of a single bunch were observed turn-by-turn. The horizontal oscillation amplitude was varied by changing the strength of the injection septum, the vertical oscillation amplitude was increased by successively reducing the injection kicker strength. The oscillation amplitudes were increased in steps until most of the beam was lost in the injection process. 12 beam position monitors (BPMs) in either plane recorded 1024 turns after the injection.

Three sets of data were taken. In each set the horizontal and vertical oscillation amplitude was varied. The first set was taken with the unchanged machine. The second set had a single sextupole (RHIC site wide name yi7-sx3) turned on with a strength of $0.09 \mathrm{~m}^{-2}$. This sextupole should drive first and third order resonances. A third data set had the sextupole sign reversed.

The sextupole that was changed in the experiment is at a location with lattice functions $\left(\beta_{x}, \beta_{y}\right)=(143 \mathrm{~m}, 50 \mathrm{~m})$. For comparison, the 72 focusing arc sextupoles are at locations $\left(\beta_{x}, \beta_{y}\right)=(45 \mathrm{~m}, 11 \mathrm{~m})$ with a strength of $+0.18 \mathrm{~m}^{-2} ; 72$ defocusing arc sextupole are at locations $\left(\beta_{x}, \beta_{y}\right)=(11 \mathrm{~m}, 44 \mathrm{~m})$ with a strength of $-0.39 \mathrm{~m}^{-2}$. The arc sextupoles correct for the natural chromaticies of $\left(\xi_{x, n}, \xi_{y, n}\right)=(-55,-57)$ and the persistent current chromaticities $\left(\xi_{x, b_{2}}, \xi_{y, b_{2}}\right)=(-38,+36)[7]$. 
Table 1: Machine and beam parameters for the experiment.

\begin{tabular}{lccc}
\hline \hline parameter & symbol & unit & value \\
\hline ring & $\ldots$ & $\ldots$ & Yellow \\
specie & $\ldots$ & $\ldots$ & $\mathrm{Au}^{79+}$ \\
relativistic beam parameter & $\gamma$ & $\ldots$ & 10.52 \\
ions per bunch & $N_{b}$ & $10^{9}$ & $0.1-0.7$ \\
normalized emittance, $95 \%$ & $\epsilon_{x, y}$ & $\mu \mathrm{m}$ & $\approx 10$ \\
tunes & $\left(\nu_{x}, \nu_{y}\right)$ & $\ldots$ & $(28.223,29.235)$ \\
chromaticities $\xi_{x, y}=\Delta v_{x, y} /(\Delta p / p)$ & $\left(\xi_{x}, \xi_{y}\right)$ & $\ldots$ & $\approx(-2,-2)$ \\
sextupole strength yi7-sx3 & $K_{2} L(\mathrm{MAD})$ & $\mathrm{m}^{-2}$ & $-0.09,0,+0.09$ \\
\hline \hline
\end{tabular}

\section{Analysis and comparison to the model}

Sextupolar fields introduce three spectral lines in the Fourier spectrum of the horizontal motion: $(-2,0),(2,0)$ and $(0,0)$. The first one is related to the third order resonance and the other two are related to the first order resonance. An example of the Fourier spectrum obtained from the experimental data is shown in Fig. 1. In this figure the tune line and the three sextupolar spectral lines are seen plus the spectral line $(-1,0)$ which is due to quadrupolar and octupolar resonances.

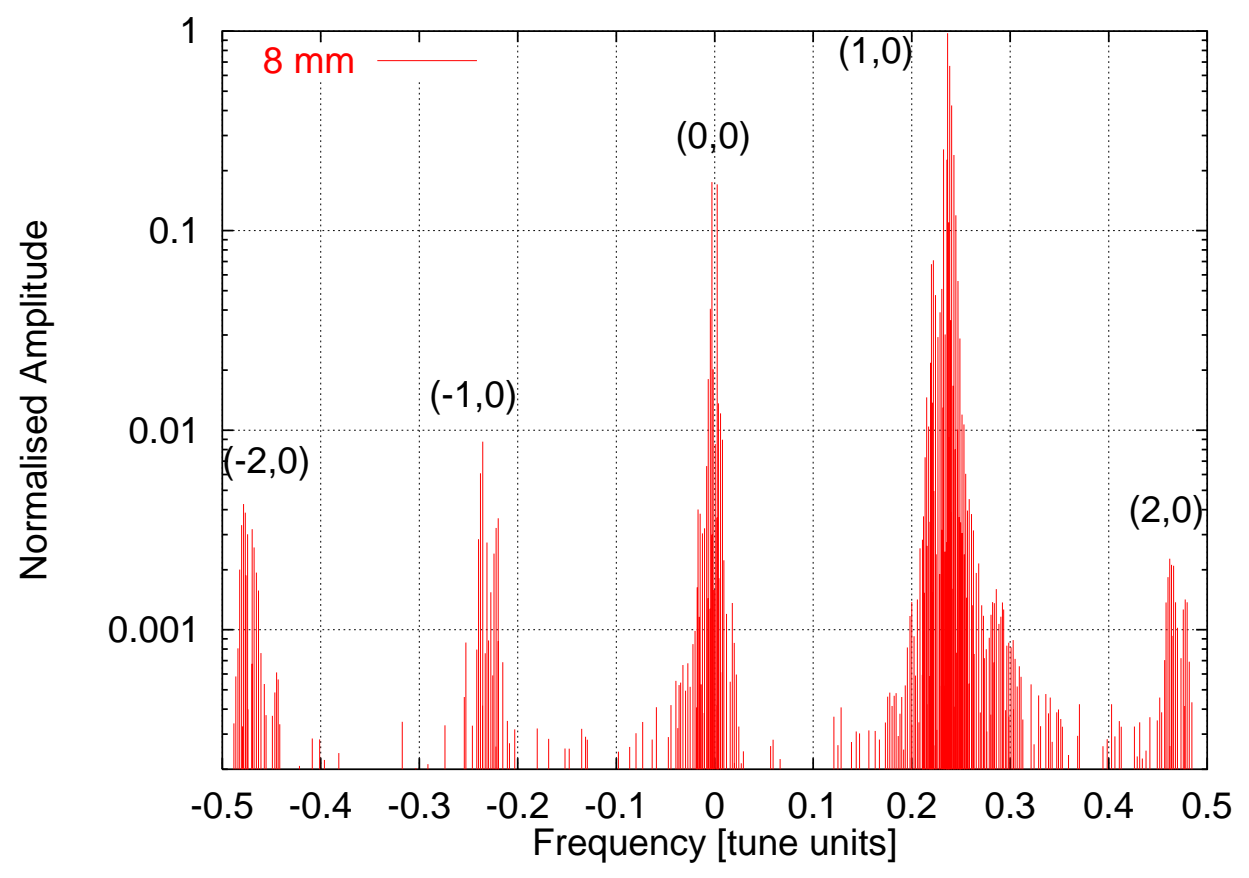

Figure 1: Fourier spectrum of the complex signal from pick-ups yo5-bh10 and yo5-bh12 for the unchanged RHIC and for an oscillation amplitude of $8 \mathrm{~mm}$. The label $(\mathrm{m}, \mathrm{n})$ means that the frequency is equal to $\mathrm{m} \nu_{x}+\mathrm{n} \nu_{y}$. 
To measure the spectral line $(0,0)$ the closed orbit previous to the excitation of the betatron motion is needed. Since in this experiment the betatron motion was excited by injecting with a certain transverse angle, the reference closed orbit is not known. Therefore the line $(0,0)$ cannot be measured.

The spectral lines $(-2,0)$ and $(2,0)$, normalised to the tune line, are proportional to the oscillation amplitude and to the resonance terms $h_{3000}$ and $h_{1200}$ respectively, as derived from Eq. (1). For the different sets of data the normalised amplitude of either spectral line is plotted versus the normalized betatron amplitude $a_{x} / \sqrt{\beta_{x}}$. The betatron amplitudes have been measured from the first turns of the BPM data. A beta function of $48 \mathrm{~m}$ has been assumed at the BPMs to compute the single particle emittance, which is twice the action $I_{x}$. Only the data coming from the pick-ups yo5-bh10 and yo5-bh12 could be systematically used for all the different settings. In Fig. 2 the normalised amplitude of the spectral line $(-2,0)$ coming from these BPMs is plotted versus the oscillation amplitude for the three different settings of the sextupole $b 2 m 07 c 3 y$. The experimental values have been multiplied by the decoherence factor of two to compare to the curves predicted by the model. The agreement is very good for the 0.0 and $0.09 \mathrm{~m}^{-2}$ cases but shows a discrepancy for the $-0.09 \mathrm{~m}^{-2}$. To measure the first order resonance the previous procedure is followed for the spectral line $(2,0)$. The resulting plots are shown in Fig. 3. Again the worst agreement is found for the case $-0.09 \mathrm{~m}^{-2}$ while for the other two sextupole settings the agreement is good.

\section{Conclusions}

For the first time we were able to demonstrate that sextupole driving terms can be measured in RHIC, an operational superconducting machine. We measured two types of horizontal sextupole resonances in a RHIC experiment and successfully compared them with model calculations of that machine.

This opens the possibility of monitoring and correcting the nonlinear content of an operational accelerator. In conjunction with an AC dipole, currently being installed in RHIC, one can use this technique continuously from injection, through the ramp, and on flat top. By using BPMs around the whole machine, one can determine driving terms as function of the longitudinal positions and identify locations of strong non-linear errors or incorrectly powered magnets.

\section{Acknowledgments}

The authors are thankful to A. Jain, S. Tepikian, and J. van Zeijts for support in the preparation of the RHIC model. F. Pilat assisted in the measurements. We are grateful to T. Satogata for his support in the data analysis. 

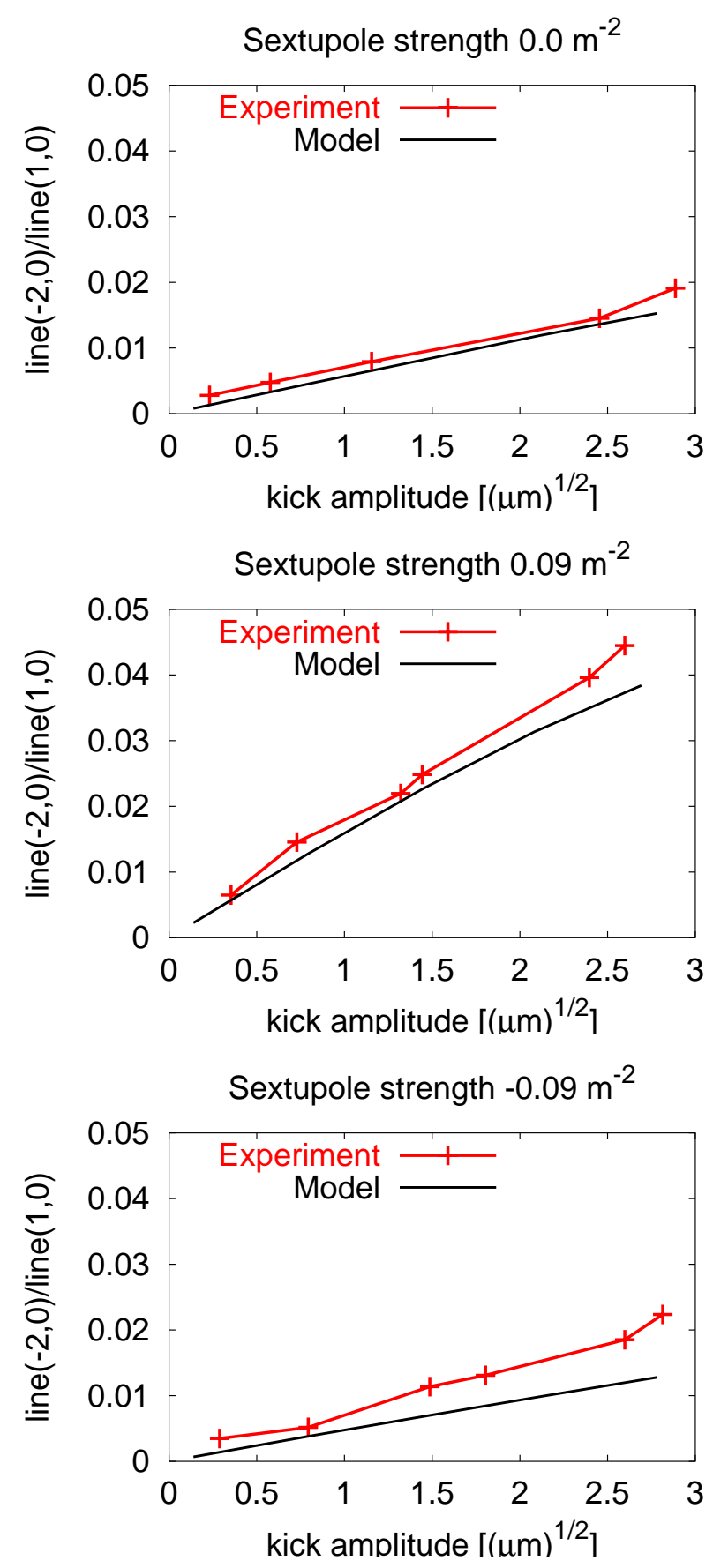

Figure 2: Measurement of the third order resonance. The normalised amplitude of the spectral line $(-2,0)$ is plotted versus horizontal betatron amplitude for three different settings of the sextupole. Experimental results are multiplied by the decoherence factor of 2 and predictions from the model are shown. 

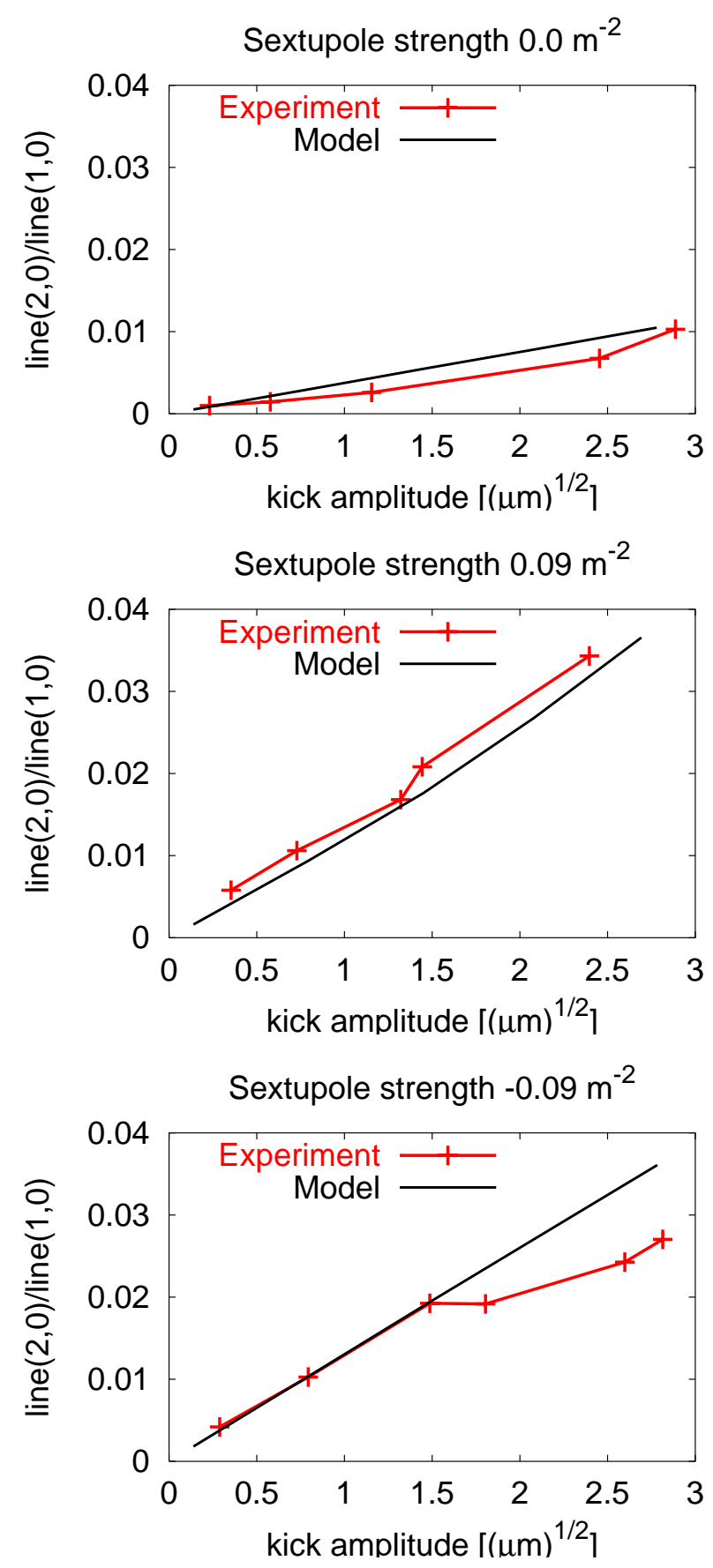

Figure 3: Measurement of the first order resonance. The normalised amplitude of the spectral line $(2,0)$ is plotted versus horizontal betatron amplitude for three different settings of the sextupole. Experimental results are multiplied by the decoherence factor of 2 and predictions from the model are shown. 


\section{References}

[1] R. Bartolini and F. Schmidt, "Normal Form via tracking or Beam Data", LHC Project note 132 (revised December 1999), Part. Accelerators. 59, pp. 93-106, (1998).

[2] F. Schmidt, R. Tomás, A. Faus-Golfe, "Measurement of Driving Terms", CERNSL-2001-039-AP Geneva, CERN , 3 Jul 2001. Pres. at: IEEE Particle Accelerator Conference, Chicago, IL, USA, 18 - 22 Jun 2001.

[3] M. Hayes, F. Schmidt, R. Tomás, "Direct Measurement of Resonance Driving Terms at SPS at $26 \mathrm{GeV",} \mathrm{Pres.} \mathrm{at:} \mathrm{8th} \mathrm{European} \mathrm{Particle} \mathrm{Accelerator} \mathrm{Conference} \mathrm{:} \mathrm{a}$ Europhysics Conference, La Vilette, Paris, France, Jun 2002 and CERN-SL-2002039-AP Geneva, 25 Jun 2002.

[4] M. Anerella et al., "The RHIC Magnet System", BNL MDN-610-20, to be published in $\operatorname{NIM}(2001)$.

[5] A. Jain, private communication (2002).

[6] F. Schmidt, "SIXTRACK, Version 3.1, Single Particle Trracking Code Treating Transverse Motion with Synchrotron Oscillations in a Symplectic Manner, User's Reference Manual", CERN SL/94-56 (AP) Update March 2000 (2000).

[7] W. Fischer, A. Jain, and S. Tepikian, "Beam-based Measurements of Persistent Current Decay in the Relativistic Heavy Ion Collider", Phys. Rev. ST Accel. Beams 4, 041002 (2001). 